\title{
Gelatin Porous Scaffolds Fabricated Using a Modified Gas Foaming Technique: Characterisation and Cytotoxicity Assessment
}

\begin{abstract}
The current study presents an effective and simple strategy to obtain stable porous scaffolds from gelatin via gas foaming method. The technique exploits the intrinsic foaming ability of gelatin in the presence of $\mathrm{CO}_{2}$ to obtain a porous structure stabilised with glutaraldehyde. The produced scaffolds were characterised using physical and mechanical characterisation methods. The results showed that gas foaming may allow the tailoring of the 3-dimensional structure of the scaffolds with an interconnected porous structure. To assess the effectiveness of preparation method in mitigating the potential cytotoxicity risk of using glutaraldehyde as a crosslinker, direct and in-direct cytotoxicity assays were performed at different concentrations of glutaraldehyde. The results indicate the potential of the gas foaming method, in the preparation of viable tissue engineering scaffolds.
\end{abstract}

Keywords: Gelatin, gas foaming, crosslinking, tensile strength, denaturation temperature;

\section{Introduction}

In tissue engineering, scaffolds provide a platform for cell attachment, migration, differentiation, and inducing a new tissue shape [1]. For the scaffold to be able to function optimally in this role, porosity is a key element. In order for the cells to penetrate beyond 500 $\mu \mathrm{m}$ from the scaffold surface, optimal porosity within the structure is necessary so that efficient nutrient transmission may take place [2]. There are multiple methods available to manufacture the 3-dimensional porous scaffolds including but not limited to: solvent casting and salt leaching [3], electro-spinning [4], and lyophilisation [5]. The major restriction of these methods is the limited thickness of the scaffold produced. 
Gas foaming is a suitable method capable of fabricating a highly porous matrix with desirable thickness. The method is based on inducing the formation of an inert gas (such as $\mathrm{CO}_{2}$ or $\mathrm{N}_{2}$ ) within a precursor solution. The formed gas transforms the liquid into a foam entity. The foam is stabilised by freezing the liquid phase with subsequent lyophilisation. Gas foaming has been used to produce porous structures from synthetic polymers, ceramic structures, and agar membranes [6-11].

In addition to the porous structure of scaffolds, great attention must be paid to their composition [5]. Gelatin is a common substrate for tissue engineering scaffold. It is a water soluble compound that traditionally is obtained from collagen hydrolysis [12-14]. Approximately 50,000 metric tons of gelatin is produced annually for medical purposes [15]. Features such as biodegradability and the possibility of cheap large-scale production allow gelatin to be an attractive candidate for the production of tissue engineering scaffolds and biomaterials [16]. From its collagen precursor, gelatin maintains peptide sequences, such as RGDs, known to promote cell adhesion [17] and migration [5,18,19]. A combination of gelatin with bioglass, biopolymers, or synthetic polymers such as poly-caprolactone showed desirable cell proliferation, and an increase in cell attachment as a result of gelatin presence in the matrix [20-22]. These features cause gelatin to be used for a variety of commercial applications in the pharmaceutical and medical fields, such as sealants for vascular prostheses $[23,24]$, bone-repairing matrices [25,26], blood plasma expanders [27,28], wound healing agents, artificial skin [29,30], and scaffolds for tissue engineering [3,31,32].

Gelatin also possesses a superior foaming ability and it is a suitable colloid stabiliser and foaming agent [33]. The food industry has taken advantage of this intrinsic feature for many years to improve the elasticity and texture of food [34-36], however there are surprisingly few investigations into the direct application of gas foaming method to prepare porous tissue engineering scaffolds and biomaterials. 
Barbetta et al. (2009) studied the feasibility of using in-situ gas foaming in gelatin solutions. A structure with high porosity and pore interconnectivity with average pore diameters of 250 to $360 \mu \mathrm{m}$ was reported, showing the potential for further investigation of this type of gelatin-based scaffolds [37]. Apart from this study, investigating the foaming application of gelatin in preparing tissue engineering scaffolds has been limited to incorporating pressurised gas into a gelatin solution to form a porous structure $[2,34]$. Pressurised foaming however, requires expensive hardware and facilities that are not readily available, and the control of processing parameters can be challenging.

In this study, the development and optimisation of a gas foaming technique was explored. Glutaraldehyde (GTA) was used to stabilise the scaffolds and to tailor their mechanical properties. GTA is a very effective crosslinking agent which functions by establishing covalent bonds with the amine groups of Lysine [38]. The GTA reaction mechanism is described as a Schiff base reaction, and establishes the carbon and nitrogen double bond $(\mathrm{C}=\mathrm{N})$ formation between GTA and gelatin molecules [39]. These reactions increase the mechanical strength and thermal stability [40], as well as life span [41] of the scaffolds. With regards to GTA toxicity, reservations in its applications have recently been raised, but there is still disagreement with regards to the extent of its cytotoxicity [41-46]. In vitro cytotoxicity was evaluated by following ISO 10993-5 guidelines with fibroblast cells to determine the effect of GTA on the biocompatibility of the prepared scaffolds. The scaffolds were further characterised using Fourier Transformed Infra-Red spectroscopy (FT-IR), mechanical tests (tensile and compression analysis), thermal analysis, and Scanning Electron Microscopy (SEM). 


\section{Materials and Methods}

\subsection{Materials}

Type B Gelatin powder from bovine skin (Bloom Index 225) was purchased from Sigma (St. Louis, MO, USA). Sodium hydrogen carbonate was procured from BDH Chemical (Poole, UK). Acetic acid and glutaraldehyde (50\% v/v) aqueous solution were both purchased from Fisher Scientific (Leicestershire, UK). All chemicals were used as received without further processing.

\subsection{Fabrication of Porous Gelatin Scaffolds}

Porous gelatin scaffolds were prepared according to the following procedure: $20 \% \mathrm{w} / \mathrm{v}$ gelatin aqueous solution was prepared by dissolving the appropriate amount of gelatin in deionised water at $50^{\circ} \mathrm{C}$. The gelatin solution was subsequently cast in stainless steel molds. Sodium hydrogen carbonate was used as the foaming agent in this study and added to molds along with the gelatin solution. Gelatin casting was shortly followed by acetic acid addition into the molds. The reaction of acetic acid with carbonate salt particles releases $\mathrm{CO}_{2}$ gas and induces the formation of the gelatin foam. The samples were transferred to a freezer $\left(-25^{\circ} \mathrm{C}\right)$ and frozen for 1 hour. The solidified samples were then removed from the metallic molds and instantly plunged into a $4^{\circ} \mathrm{C}$ water bath to extract traces of unreacted compounds. Crosslinked gelatin scaffolds were prepared by immersion in $0.25,0.50,0.75$, and $1.00 \% \mathrm{v} / \mathrm{v}$ GTA solutions for 3 hours. Control samples were left non-crosslinked for comparison purposes. Samples were then washed with de-ionised water overnight to remove traces of GTA. The samples were frozen and then lyophilised for 24 hours. 


\subsection{Characterisation of the Prepared Scaffolds}

\subsubsection{FT-IR Spectroscopy}

The gelatin macromolecular structure and potential impact of crosslinking on the gelatin scaffolds were characterised using Fourier Transformed Infra-Red spectroscopy (FTIR/ATR-4800s, Shimadzu, Japan). All spectra were obtained by scanning from 4000 to $1000 \mathrm{~cm}^{-1}$ at a nominal resolution of $4 \mathrm{~cm}^{-1}$ using 264 scans. The obtained results were normalised against a background scan collected at ambient temperature. The samples were kept in a desiccator with self-indicating silica gel prior to analysis for 48 hours.

\subsubsection{Thermal Analysis}

In order to investigate the effect of crosslinking on the denaturation temperature of the gelatin scaffolds, thermal analysis was performed using a Differential Scanning Calorimetry (DSC 822e, Mettler-Toledo, Switzerland). All samples were conditioned for 48 hours at $65 \%$ relative humidity and $20^{\circ} \mathrm{C}$ prior to analysis. The samples were placed in $40 \mu \mathrm{l}$ aluminum pans and hermetically sealed. The samples were heated from 15 to $100^{\circ} \mathrm{C}$ at a heating rate of $5^{\circ} \mathrm{C} \cdot \mathrm{min}^{-1}$. The peak temperature and the normalised enthalpy of transition of each sample were recorded. The normalised enthalpy of transition was computed as the integrated area under the transition peak. The transition peak temperature was assigned as the denaturation temperature $\left(\mathrm{T}_{\mathrm{d}}\right)$.

\subsubsection{Mechanical Tests}

\subsubsection{Compression Tests}

All samples were conditioned for 48 hours at $95 \%$ relative humidity and $20^{\circ} \mathrm{C}$. The samples were examined in compression and tensile modes using a TA.XT-Plus texture analyzer (Stable Micro Systems, Surrey, UK). To perform the compression tests, the method 
described by Leffler and Muller (2000) was applied to measure the scaffolds compression strength [11]. Briefly, the samples were compressed to $40 \%$ of their initial height with a crosshead speed of $2 \mathrm{~mm} \cdot \mathrm{s}^{-1}$ and the scaffold resistance was recorded as the peak force $(\mathrm{N})$. The recorded force was then used to compute the scaffolds compression strength using Equation 1, where $\sigma$ is the scaffold's compression strength $(\mathrm{Pa}), \mathrm{F}$ is the peak force $(\mathrm{N}), \mathrm{A}_{0}$ is the theoretical cross section area $\left(\mathrm{m}^{2}\right)$.

$$
\left.\sigma=\frac{F}{A_{0}} \quad \text { (Equation } 1\right)
$$

\subsubsection{Tensile Strength Tests}

Uni-axial tensile tests were performed in order to further characterise the mechanical properties of the scaffolds. The sample conditioning criteria were as described in Section 2.3.3.1. The samples were cut into rectangular strips $(10 \times 5 \mathrm{~mm})$. The thickness of the samples was measured at 3 points using digital callipers and their average value was used for calculating the cross section surface area. The samples were drawn with a cross head speed of $0.033 \mathrm{~mm} . \mathrm{s}^{-1}$ until sample rupture was detected. The rupture force was recorded to measure the sample tensile strength using Equation 1. Tensile properties were calculated from a Stress-Strain plot. The scaffolds tensile strain was calculated using Equation 2; where $\varepsilon$ is the tensile strain (\%), $\delta$ is the change in gauge length at the sample rupture point $(\mathrm{mm})$, and $\mathrm{L}_{0}$ is the initial gauge length $(\mathrm{mm})$. The scaffold elastic modulus was calculated as the gradient of the linear segment of the Stress-Strain plot and is expressed in $\mathrm{kPa}$.

$$
\varepsilon=\frac{\delta}{L_{0}} \times 100 \quad \text { (Equation 2) }
$$




\subsubsection{Microstructure Analysis}

The lyophilised scaffolds were kept in a desiccator containing self-indicating silica gel prior to examination for 48 hours. The scaffolds were cross-sectioned using a surgical scalpel and examined using a Scanning Electron Microscope (SEM). Samples were gold-coated using a sputter coater (Mscope, SC500, UK) and their structural morphology examined at an accelerating voltage of $5 \mathrm{kV}$ (Hitachi Variable Pressure Scanning Electron Microscope, S3000N, Japan).

\subsubsection{Cytotoxicity Analysis}

Different concentrations of GTA $(0.25,0.50,0.75$, and $1.00 \% \mathrm{v} / \mathrm{v})$ were used to crosslink the prepared scaffolds and tested according to ISO 10993-5 guidelines for direct and indirect cytotoxicity according to the method described elsewhere [47]. Before all analysis, samples were sterilised under UV light for 6 hours.

For indirect tests, scaffolds $(3 \times 3 \times 5 \mathrm{~mm})$ were incubated in triplicate in $2 \mathrm{ml}$ Dulbecco's Modified Eagle's Medium (DMEM, Gibco, Life Technologies, UK) with 10\% v/v of Fetal Bovine Serum (FBS, Gibco, Life Technologies, UK) in $50 \mathrm{ml}$ Falcon tubes (BD Bioscience, USA) at $37^{\circ} \mathrm{C}, 5 \% \mathrm{CO}_{2}$, and fully humidified air for 3 days. The resulting medium, enriched in lixiviates potentially released from the tested materials, were used to culture L929 mouse fibroblasts cell line (DSMZ, Germany) with an initial density of $80 \times 10^{3}$ cells $/ \mathrm{cm}^{2}$ on 24 -well plates for 3 days. Metabolic activity of cultured cells was determined using a MTT (3-[4,5dimethylthiazol-2-yl]-2,5-diphenyl tetrazolium bromide) cell proliferation kit (Sigma Aldrich, USA). The results were normalised to the negative control for cytotoxicity analysis (fresh DMEM with 10\% v/v FBS medium) and compared to the positive control medium preincubated with latex. 
For the direct contact assays, L929 cells were seeded at a density of $80 \times 10^{3}$ cells $/ \mathrm{cm}^{2}$ on 6-well plates (BD Bioscience, USA) until confluence was reached; the scaffolds were gently placed directly above the cultured cells (2 ml DMEM with 10\% v/v FBS). The cultures were incubated at $37^{\circ} \mathrm{C}, 5 \% \mathrm{CO}_{2}$, in fully humidified air for 3 days. The cells were then visualised using an inverted microscope (Leica Microsystems, Germany) to determine if an inhibitory halo at the scaffold interface formed. The control was a culture of L929 cells cultivated on a tissue culture polystyrene 6-well plate, without contact with the scaffolds.

\subsubsection{Statistical Analysis}

The presence of significant differences between results was verified using nonparametric tests according to Kruskal-Wallis method using SPSS Statistics (Version 20) software (International Business Machine, NY, USA). Differences between results were analysed using Mann-Whitney test method and accepted as significant at $\mathrm{p} \leq 0.05$. 


\section{Results and Discussion}

\subsection{FT-IR Spectroscopy}

Figure 1 compares the FT-IR spectra of scaffolds crosslinked with $0,0.25,0.50,0.75$, and $1.00 \%$ v/v GTA. The spectra of all 5 samples exhibited the amide characteristic peaks of gelatin at 1627,1548 , and $1234 \mathrm{~cm}^{-1}$. Absorptions at $1627 \mathrm{~cm}^{-1}$ correspond to the amide I band which is primarily due to the stretching vibrations of peptide carbonyl groups [48-51]. Amide II absorption band at $1548 \mathrm{~cm}^{-1}$ is due to $\mathrm{N}-\mathrm{H}$ bending and $\mathrm{N}=\mathrm{C}$ stretching [51], and amide III band absorption occurred at $1234 \mathrm{~cm}^{-1}$ due to weak $\mathrm{N}=\mathrm{C}$ stretching and $\mathrm{N}-\mathrm{H}$ bending [50]. All samples displayed amide A and amide B bands located in the region between 3290 and $3060 \mathrm{~cm}^{-1}$, respectively. These two bands are assigned to the N-H stretching vibration [50,52]. The C-H stretching modes were also found between 2900 and $3000 \mathrm{~cm}^{-1}[53]$.

The spectra of the crosslinked samples show a slight shift towards lower wave numbers in the amide I and III bands relative to non-crosslinked samples. This shift may be due to structural changes caused by crosslinking [48,52]. Farris et al. reported a similar shift towards lower wave numbers as result of crosslinking gelatin with GTA at an acidic $\mathrm{pH}$. It was suggested that this shift may be due to crosslinking of gelatin molecules [54]. The results may be indicative of subtle structural changes due to the GTA reaction with gelatin macromolecules but reaffirm that crosslinking did not substantially alter the gelatin macromolecular structure.

\subsection{Thermal Analysis}

Table 1 lists thermal characteristics of the non-crosslinked and crosslinked scaffolds at various concentrations of GTA. Denaturation temperature $\left(T_{d}\right)$ is an indirect measurement of the degree of crosslinking: the higher is the $T_{d}$ value, the greater the degree of crosslinking 
[55]. A gradual increase in the gelatin denaturation temperature $\left(T_{d}\right)$ occurred as the GTA concentration increased. Non-crosslinked samples showed a thermal transition with a $T_{d}$ at $44^{\circ} \mathrm{C}$. The $\mathrm{T}_{\mathrm{d}}$ of the crosslinked samples were found to be between 74 and $78^{\circ} \mathrm{C}$. The formation of crosslink bonds between the gelatin molecules is responsible for an increase in the denaturation temperature [41]. This is thought to be due to a decrease in the entropy of transition [56]. Along with an increase in $T_{d}$, the enthalpy of transition $(\Delta H)$, normalised with respect to sample weight, decreased as crosslinker concentration increased. Reduction in enthalpy is caused by a reduction in the number of hydrogen bonds in favour of an increase in the number of covalent bonds. Covalent bonds break exothermically whilst hydrogen bonds break endothermically $[41,57,58]$. The normalised enthalpy of transition was reduced from $14.22 \mathrm{~J} / \mathrm{g}$ in the non-crosslinked samples to $-9.65 \mathrm{~J} / \mathrm{g}$ in the scaffolds crosslinked with $1.00 \%$ v/v GTA solution (Table 1).

\subsection{Mechanical Properties}

Table 2 lists the mechanical properties of gelatin scaffolds in compression mode. The compression strength of the prepared samples increased as the GTA concentration increased. As listed in Table 2, Compression strength of scaffolds crosslinked with $1.00 \% \mathrm{v} / \mathrm{v}$ GTA solution was 4 times higher as compared with the non-crosslinked samples. Although the addition of GTA to the structure increased the compression strength, no significant differences were found between compression strength of non-crosslinked samples and scaffolds crosslinked below $0.75 \%$ v/v GTA $(\mathrm{p}=0.40)$. The compression strength significantly increased when the GTA concentration increased above $0.75 \% \mathrm{v} / \mathrm{v}(\mathrm{p}=0.03)$. Increase of the GTA concentration above $1.00 \%$ may cause a further increase in the compression strength, however this study is beyond the scope of this article and can be investigated in future studies. 
Table 3 lists the tensile properties of the prepared scaffolds. The tensile strength of the scaffolds increased with GTA concentration up to $0.50 \%$ v/v GTA solution. Crosslinking with concentrations above $0.50 \% \mathrm{v} / \mathrm{v}$ resulted in the tensile strength to decrease. The initial increase of the tensile strength was statistically significant for GTA concentrations $0.50 \% \mathrm{v} / \mathrm{v}$ in comparison with the non-crosslinked samples $(\mathrm{p}=0.02)$.

An arrest of increase in tensile strength upon further increase of GTA concentration above $0.50 \%$ may have been due to complete crosslinking of the gelatin structure and a combination of increased structural stiffness and irregular porosity in the scaffolds. Wu et al. (2010) reported that a decrease of the tensile strength at high concentrations of GTA may be due to the over-crosslinking of the gelatin structure. Other studies have also shown a decrease of the gelatin mechanical strength as a result of higher crosslinker concentrations $[14,59,60]$. Knaul et al. (1999) suggested that the decrease in the mechanical strength at high concentrations of GTA may be due to: degradation of molecular structure at relatively high concentrations, local stress concentrations, and a decrease in crystallinity upon drying with increasing crosslinking density [40]. Irregular porosity has been suggested as a source of structural defects contributing to elevated local stress at the pore edges, leading to a reduced mechanical resistance against crack initiation [61]. An increase in GTA concentration causes the specimen to become less plastic [14,62], which showed less resistance against crack initiation and this led to a decrease in tensile strength in scaffolds crosslinked with 0.75 and $1.00 \%$ v/v GTA aqueous solution.

There is a significant difference between the elastic modulus of non-crosslinked samples and crosslinked samples up to and including the GTA concentration of $0.50 \% \mathrm{v} / \mathrm{v}$ $(\mathrm{p}=0.01)$. This is mainly due to effect of crosslinking on the tensile strain of the scaffolds and is in agreement with the results reported for crosslinked gelatin [63]. 
Crosslinking decreased the tensile strain of the gelatin scaffolds. The non-crosslinked samples showed the lowest tensile strain when compared with the crosslinked samples, this may be due to poor sample integrity causing the structure to fail prematurely during tensile testing and therefore true measurement of the scaffold properties is difficult. In comparison with the non-crosslinked sample, the decrease of the tensile strain in crosslinked scaffolds was significant for the scaffolds crosslinked with GTA concentrations above $0.25 \%(\mathrm{p}=0.02)$. The tensile strain was inversely proportional to increased GTA concentration except for the low concentration of $0.25 \% \mathrm{v} / \mathrm{v}$ (Table 3 ).

\subsection{Microstructure Analysis}

Figure 2 (A-F) shows the SEM images of the non-crosslinked samples and scaffolds crosslinked with 0.50 and $1.00 \%$ v/v GTA solution. Varying the GTA concentration affected both the pore size and textural structure of the scaffolds. In general, the non-crosslinked samples (Figure 2-A and B) showed a more flat and smoother surface as compared with the crosslinked scaffolds shown in Figure 2-C to F. In particular, the structure in the noncrosslinked samples appeared to have collapsed with less interconnectivity, whilst the crosslinked structures showed more pore interconnectivity. As shown in Figure 2-B, the pores in the non-crosslinked samples showed signs of distortion which may be due to lack of mechanical strength.

The average pore size in scaffolds crosslinked with $0.50 \%$ v/v GTA solution (Figure 2C and D) was $280 \mu \mathrm{m}$. Scaffolds crosslinked with $1.00 \% \mathrm{v} / \mathrm{v}$ GTA showed increased pore interconnectivity when compared with the flat structure of non-crosslinked scaffolds (Figure 2-A and E). The microstructure of the scaffolds crosslinked with $1.00 \%$ v/v GTA solution contained larger pores in comparison with samples prepared with $0.50 \%$ v/v GTA (Figures 2E and F). The average pore size in $1.00 \% \mathrm{v} / \mathrm{v}$ GTA crosslinked scaffolds was $550 \mu \mathrm{m}$. The 
thickness of the pore walls varied between $30 \mu \mathrm{m}$ (in the area between two adjacent pores) and $170 \mu \mathrm{m}$ around vertices where three or more pores converged with each other (Figure 2F). This showed a reduction of the pore size when compared with the scaffolds crosslinked at $1.00 \% \mathrm{v} / \mathrm{v}$ GTA concentration (Figure 2-E and F). Crosslinking with GTA may improve the stability of the porous microstructure within the gelatin matrix. Higher concentration of GTA may have led to more effective crosslinking and better preservation of porous structure during the synthesis process, as the gelatin foam stabilization occurred more rapidly, preserving the porous nature of the original gelatin foam.

\subsection{Cytotoxicity Evaluation}

\subsubsection{Indirect Cytotoxicity Evaluation}

Gelatin is a commonly used material for tissue engineering scaffolds. To improve scaffold stability and to produce a highly porous structure, GTA was used as a crosslinking agent in this study. Due to the nature of unreacted aldehyde groups, application of GTA may be incompatible with cell viability. Therefore the scaffolds were tested for cytotoxicity using L929 cells following the ISO 10993-5 guidelines for indirect and direct contact tests [47]. The results indicated that a slight increase in cytotoxicity with increasing GTA concentration occurred in the indirect tests (Figure 3). The indirect tests assess the toxicity of lixiviates that leach into the cell culture medium during the 3 day incubation period, either due to the residual GTA within the scaffolds or from potential biotransformation and degradation at $37^{\circ} \mathrm{C}$. MTT assays were used to assess the effects on cell activity of lixiviates in the cell culture media. The results showed a decrease in cell activity for media lixiviates from scaffolds with increasing GTA concentrations used for crosslinking. This highlights a possible increase in the toxicity of the scaffolds corresponding with the degree of GTA crosslinking. However, cell activity for all the lixiviate samples was significantly well above 
the positive control and approximately $79 \%$ or higher of the negative control (Figure 3). These results indicate that the potential toxicity of lixiviates from the tested materials was low enough to proceed to the direct contact tests.

\subsubsection{Direct Contact Evaluation}

The direct contact tests were carried out by incubating L929 mouse fibroblasts cells and the scaffolds in the same culture wells for 3 days, in order to observe if the material is responsible for any morphological changes and/or cell death. The negative control consisted of cells cultured in a common culture dish in the absence of contact with the sample. It should be noted that for the non-crosslinked scaffolds, the structure disintegrated during the 3 day culture period due to lack of crosslinking. All scaffolds appeared to be nontoxic towards the cells in direct contact, regardless of the GTA concentrations used, since no halo of inhibition was observed in the vicinity of sample edges (Figure 4). Since inverted microscopy was used and the porous gelatin scaffolds were slightly translucent at the edges, the projection of the cells beneath the scaffolds with a regular morphology of healthy fibroblasts was visible.

\section{Summary}

In this study, the feasibility of using gas foaming method to produce gelatin foam blocks was shown and the effect of crosslinking on the structure was investigated. The microstructure of the crosslinked scaffolds showed desirable inter-connected porosity. Pore size and topography of gelatin structure were altered with increasing GTA concentration. Higher GTA concentrations produced a larger pore size, as evaluated with SEM. Tensile and compression strength of the scaffolds were affected as result of GTA crosslinking. The compression strength of the scaffolds increased with GTA concentration, whilst the tensile strength of structures increased up to $0.50 \% \mathrm{v} / \mathrm{v}$ GTA and decreased subsequently due to the porous structure and reduced elasticity. Crosslinked scaffolds did not show any sign of 
cytotoxicity in direct contact tests and cell activity remained above $80 \%$ of negative control in indirect assays. It should be pointed out that this study is not aimed at investigating the direct effect of GTA on the cell metabolism, but merely verifies if the synthesising procedure used in this study and washing out that took place after crosslinking the scaffolds was effective enough to extract most of the remaining GTA compound left in the samples and rendered the scaffolds cell friendly. Increase of cytotoxicity at the highest concentration of GTA showed that overnight washing of the sample was not adequate. To mitigate this concern neutralising unreacted GTA compound via Glycerol washout can be recommended. However, considering reported toxicity of GTA, it is suggested that alternative crosslinking agents be considered in future studies. Considering the simplicity and affordability of the reported synthesis method, the results of this study suggest that the gas foaming may be explored further as a potential method for manufacturing tissue engineering scaffolds. Low mechanical strength of the scaffolds may be a concern in the field of tissue engineering. To address this weakness the following improvements may be recommended: (1) modification of the gas foaming method to reduce the pore size so that more desirable tensile strength can be achieved, and (2) addition of components with higher tensile strength such as bioceramics and bioglasses which are reported to be effective in improving the tensile strength of the gelatin structure [20,64].

\section{Acknowledgements}

This work was financially supported by the Fundação para a Ciência e Tecnologia (FCT) through project PTDC/EQU-EQU/114231/2009 and MIT-Portugal Program, Bioengineering Systems Focus Area. JH and FCF acknowledge FCT for granting the PhD scholarship SFRH/BD/61450/2009 and the research contract IF/00442/2012. The Authors wish to acknowledge financial support provided by University of Northampton Chancellor Fund Trustees, and Armourers \& Brasiers' Gauntlet Trust, UK. The authors express sincere gratitude to Ms. Leticia Melo Dos Santos for assistance with revision of this manuscript. 


\section{References}

[1] S. Natesan, P. Ravichandran, P. Neelakanta Reddy, N. Ramamurty, S. Pal, K. Panduranga Rao, Collagen-chitosan polymeric scaffolds for the in vitro culture of human epidermoid carcinoma cells, Biomaterials. 22 (2001) 1943-1951.

[2] F. Dehghani, N. Annabi, Engineering porous scaffolds using gas-based techniques, Curr. Opin. Biotechnol. 22 (2011) 661-666.

[3] S.B. Lee, Y.H. Kim, M.S. Chong, S.H. Hong, Y.M. Lee, Study of gelatin-containing artificial skin V: fabrication of gelatin scaffolds using a salt-leaching method, Biomaterials. 26 (2005) 1961-1968.

[4] N. Charernsriwilaiwat, T. Rojanarata, T. Ngawhirunpat, P. Opanasopit, Electrospun chitosan/polyvinyl alcohol nanofibre mats for wound healing, International Wound Journal. 452 (2013) 333-343.

[5] S. Moscato, L. Mattii, D. D’Alessandro, M.G. Cascone, L. Lazzeri, L.P. Serino, A. Dolfi, N. Bernardini, Interaction of human gingival fibroblasts with PVA/gelatine sponges, Micron. 39 (2008) 569-579.

[6] A. Almirall, G. Larrecq, J.A. Delgado, S. Martínez, J.A. Planell, M.P. Ginebra, Fabrication of low temperature macroporous hydroxyapatite scaffolds by foaming and hydrolysis of an $\alpha$-TCP paste, Biomaterials. 25 (2004) 3671-3680.

[7] A. Nussinovitch, Mechanical properties of hydrocolloid gels filled with internally produced carbon dioxide gas bubbles, Biotechnol. Prog. 8 (1992) 424-428.

[8] J.J. Yoon, T.G. Park, Degradation behaviors of biodegradable macroporous scaffolds prepared by gas foaming of effervescent salts, J. Biomed. Mater. Res. 55 (2001) 401-408.

[9] Y.S. Nam, J.J. Yoon, T.G. Park, A novel fabrication method of macroporous biodegradable polymer scaffolds using gas foaming salt as a porogen additive, J. Biomed. Mater. Res. 53 (2000) 1-7.

[10] S. Hesaraki, A. Zamanian, F. Moztarzadeh, The influence of the acidic component of the gas-foaming porogen used in preparing an injectable porous calcium phosphate cement on its properties: Acetic acid versus citric acid, J. Biomed. Mater. Res. B. 86B (2008) 208-216.

[11] C.C. Leffler, B.W. Müller, Influence of the acid type on the physical and drug liberation properties of chitosan-gelatin sponges, Int. J. Pharm. 194 (2000) 229-237.

[12] S. Ghoshal, C. Mattea, S. Stapf, Inhomogeneity in the drying process of gelatin film formation: NMR microscopy and relaxation study, Chem. Phys. Lett. 485 (2010) 343-347.

[13] A. Bigi, S. Panzavolta, K. Rubini, Relationship between triple-helix content and mechanical properties of gelatin films, Biomaterials. 25 (2004) 5675-5680. 
[14] A. Bigi, G. Cojazzi, S. Panzavolta, K. Rubini, N. Roveri, Mechanical and thermal properties of gelatin films at different degrees of glutaraldehyde crosslinking, Biomaterials. 22 (2001) 763-768.

[15] D. Olsen, C. Yang, M. Bodo, R. Chang, S. Leigh, J. Baez, D. Carmichael, M. Perälä, E. Hämäläinen, M. Jarvinen, J. Polarek, Recombinant collagen and gelatin for drug delivery, Adv. Drug Deliv. Rev. 55 (2003) 1547-1567.

[16] T.J. Koob, D.J. Hernandez, Mechanical and thermal properties of novel polymerized NDGA-gelatin hydrogels, Biomaterials. 24 (2003) 1285-1292.

[17] B. Kim, I. Park, T. Hoshiba, H. Jiang, Y. Choi, T. Akaike, C. Cho, Design of artificial extracellular matrices for tissue engineering, Progress in Polymer Science. 36 (2011) 238268.

[18] H. Hajiali, S. Shahghasempour, M.R. Naimi-Jamal, H. Peirovi, Electrospun PGA/gelatin nanofibrous scaffolds and their potential application in vascular tissue engineering, Int. J. Nanomed. 6 (2011) 2133-2141.

[19] S.C.C.C. Miranda, G.A.B. Silva, R.C.R. Hell, M.D. Martins, J.B. Alves, A.M. Goes, Three-dimensional culture of rat BMMSCs in a porous chitosan-gelatin scaffold: A promising association for bone tissue engineering in oral reconstruction, Arch. Oral Biol. 56 (2011) 1-15.

[20] P. Gentile, M. Mattioli-Belmonte, V. Chiono, C. Ferretti, F. Baino, C. Tonda-Turo, C. Vitale-Brovarone, I. Pashkuleva, R.L. Reis, G. Ciardelli, Bioactive glass/polymer composite scaffolds mimicking bone tissue, J. Biomed. Mater. Res. A. 100A (2012) 2654-2667.

[21] Y. Huang, S. Onyeri, M. Siewe, A. Moshfeghian, S.V. Madihally, In vitro characterization of chitosan-gelatin scaffolds for tissue engineering, Biomaterials. 26 (2005) 7616-7627.

[22] J. Lee, J.J. Yoo, A. Atala, S.J. Lee, Controlled heparin conjugation on electrospun poly( $\varepsilon$-caprolactone)/gelatin fibers for morphology-dependent protein delivery and enhanced cellular affinity, Acta Biomater. 8 (2012) 2549-2558.

[23] S. Young, M. Wong, Y. Tabata, A.G. Mikos, Gelatin as a delivery vehicle for the controlled release of bioactive molecules, J. Controlled Release. 109 (2005) 256-274.

[24] C.M. Elvin, T. Vuocolo, A.G. Brownlee, L. Sando, M.G. Huson, N.E. Liyou, P.R. Stockwell, R.E. Lyons, M. Kim, G.A. Edwards, G. Johnson, G.A. McFarland, J.A.M. Ramshaw, J.A. Werkmeister, A highly elastic tissue sealant based on photopolymerised gelatin, Biomaterials. 31 (2010) 8323-8331.

[25] M. Azami, A. Samadikuchaksaraei , A. Poursamar, Synthesis and characterization of a laminated hydroxyapatite/gelatin nanocomposite scaffold with controlled pore structure for bone tissue engineering, Int. J. Artif. Organs. 33 (2010) 86-95. 
[26] P. Gentile, V. Chiono, F. Boccafoschi, F. Baino, C. Vitalo-Brovarone, E. Verne, N. Barbani, G. Ciardelli, Hydroxyapatite/bioactive glass for tissue engineering applications, J. Biomater. Sci. Polymer Edn. 21 (2010) 1207-1226.

[27] Z. Dong, Q. Wang, Y. Du, Alginate/gelatin blend films and their properties for drug controlled release, J. Membr. Sci. 280 (2006) 37-44.

[28] Y.S. Choi, S.R. Hong, Y.M. Lee, K.W. Song, M.H. Park, Y.S. Nam, Study on gelatincontaining artificial skin: I. Preparation and characteristics of novel gelatin-alginate sponge, Biomaterials. 20 (1999) 409-417.

[29] S.E. Noorjahan, T.P. Sastry, An in vivo study of hydrogels based on physiologically clotted fibrin-gelatin composites as wound-dressing materials, J. Biomed. Mater. Res. B. 71B (2004) 305-312.

[30] S.R. Hong, S.J. Lee, J.W. Shim, Y.S. Choi, Y.M. Lee, K.W. Song, M.H. Park, Y.S. Nam, S.I. Lee, Study on gelatin-containing artificial skin IV: a comparative study on the effect of antibiotic and EGF on cell proliferation during epidermal healing, Biomaterials. 22 (2001) 2777-2783.

[31] M. Yeh, Y. Liang, K. Cheng, N. Dai, C. Liu, J. Young, A novel cell support membrane for skin tissue engineering: Gelatin film cross-linked with 2-chloro-1-methylpyridinium iodide, Polymer. 52 (2011) 996-1003.

[32] J. Mao, L. Zhao, K. de Yao, Q. Shang, G. Yang, Y. Cao, Study of novel chitosan-gelatin artificial skin in vitro, J. Biomed. Mater. Res. A. 64A (2003) 301-308.

[33] M.C. Gómez-Guillén, B. Giménez, M.E. López-Caballero, M.P. Montero, Functional and bioactive properties of collagen and gelatin from alternative sources: A review, Food Hydrocoll. 25 (2011) 1813-1827.

[34] R.N. Zuniga, J.M. Aguilera, Structure-fracture relationships in gas-filled gelatin gels, Food Hydrocoll. 23 (2009) 1351-1357.

[35] J.M. Koli, S. Basu, B.B. Nayak, S.B. Patange, A.U. Pagarkar, V. Gudipati, Functional characteristics of gelatin extracted from skin and bone of Tiger-toothed croaker (Otolithes ruber) and Pink perch (Nemipterus japonicus), Food Bioprod. Process. 90 (2012) 555-562.

[36] R. Jeya Shakila, E. Jeevithan, A. Varatharajakumar, G. Jeyasekaran, D. Sukumar, Functional characterization of gelatin extracted from bones of red snapper and grouper in comparison with mammalian gelatin, LWT - Food Science and Technology. 48 (2012) 30-36.

[37] A. Barbetta, A. Gumerio, R. Pecci, R. Bedini, M. Dentini, Gas-in-Liquid Foam Templating as a Method for the Production of Highly Porous Scaffolds, Biomacromolecules. 10 (2009) 3188-3192.

[38] V. Charulatha, A. Rajaram, Influence of different crosslinking treatments on the physical properties of collagen membranes, Biomaterials. 24 (2003) 759-767. 
[39] L.H.H. Damink, P.J. Dijkstra, J.A.V. Luyn, P.B.V. Wachem, P. Nieuwenhuis, J. Feijen, Glutaraldehyde as crosslinking agent for collagen-based biomaterials, J. Mater. Sci. Mater. M. 6 (1995) 460-472.

[40] J.Z. Knaul, S.M. Hudson, K.A.M. Creber, Crosslinking of chitosan fibers with dialdehydes: Proposal of a new reaction mechanism, J. Biomed. Mater. Res. B. 37 (1999) 1079-1094.

[41] R.A. De Carvalho, C.R.F. Grosso, Characterization of gelatin based films modified with transglutaminase, glyoxal and formaldehyde, Food Hydrocoll. 18 (2004) 717-726.

[42] W. Ma, C. Tang, S. Yin, X. Yang, J. Qi, Genipin-crosslinked gelatin films as controlled releasing carriers of lysozyme, Food Res. Int. 51 (2013) 321-324.

[43] H. Sung, I. Liang, C. Chen, R. Huang, H. Liang, Stability of a biological tissue fixed with a naturally occurring crosslinking agent (genipin), J. Biomed. Mater. Res. 55 (2001) 538-546.

[44] G. Speit, S. Neuss, P. Schütz, M. Fröhler-Keller, O. Schmid, The genotoxic potential of glutaraldehyde in mammalian cells in vitro in comparison with formaldehyde, Mutat. Res. Gen. Tox. En. 649 (2008) 146-154.

[45] E. Khor, Methods for the treatment of collagenous tissues for bioprostheses, Biomaterials. 18 (1997) 95-105.

[46] T.J. Koob, T.A. Willis, Y.S. Qiu, D.J. Hernandez, Biocompatibility of NDGApolymerized collagen fibers. II. Attachment, proliferation, and migration of tendon fibroblasts in vitro, J. Biomed. Mater. Res. 56 (2001) 40-48.

[47] M. Temtem, L.M.C. Silva, P.Z. Andrade, F. dos Santos, C.L. da Silva, J.M.S. Cabral, M.M. Abecasis, A. Aguiar-Ricardo, Supercritical CO2 generating chitosan devices with controlled morphology. Potential application for drug delivery and mesenchymal stem cell culture, J. Supercrit. Fluid. 48 (2009) 269-277.

[48] K.J. Payne, A. Veis, Fourier transform IR spectroscopy of collagen and gelatin solutions: Deconvolution of the amide I band for conformational studies, Biopolymers. 27 (1988) 17491760 .

[49] D.M. Hashim, Y.B.C. Man, R. Norakasha, M. Shuhaimi, Y. Salmah, Z.A. Syahariza, Potential use of Fourier transform infrared spectroscopy for differentiation of bovine and porcine gelatins, Food Chem. 118 (2010) 856-860.

[50] A.A. Haroun, S.A. El Toumy, Effect of natural polyphenols on physicochemical properties of crosslinked gelatin-based polymeric biocomposite, J Appl Polym Sci. 116 (2010) 2825-2832.

[51] M. Jackson, L. Choo, P.H. Watson, W.C. Halliday, H.H. Mantsch, Beware of connective tissue proteins: Assignment and implications of collagen absorptions in infrared spectra of human tissues, Biochimica et Biophysica Acta (BBA) - Molecular Basis of Disease. 1270 (1995) 1-6. 
[52] J.H. Muyonga, C.G.B. Cole, K.G. Duodu, Fourier transform infrared (FTIR) spectroscopic study of acid soluble collagen and gelatin from skins and bones of young and adult Nile perch (Lates niloticus), Food Chem. 86 (2004) 325-332.

[53] H. Susi, J.S. Ard, R.J. Carroll, The infrared spectrum and water binding of collagen as a function of relative humidity, Biopolymers. 10 (1971) 1597-1604.

[54] S. Farris, J. Song, Q. Huang, Alternative reaction mechanism for the cross-linking of gelatin with glutaraldehyde, J. Agric. Food. Chem. 58 (2010) 998-1003.

[55] P.F. Gratzer, C.A. Pereira, J.M. Lee, Solvent environment modulates effects of glutaraldehyde crosslinking on tissue-derived biomaterials, J. Biomed. Mater. Res. 31 (1996) 533-543.

[56] R. Usha, T. Ramasami, Effect of crosslinking agents (basic chromium sulfate and formaldehyde) on the thermal and thermomechanical stability of rat tail tendon collagen fibre, Thermochim. Acta. 356 (2000) 59-66.

[57] D. Achet, X.W. He, Determination of the renaturation level in gelatin films, Polymer. 36 (1995) 787-791.

[58] G. Dardelle, A. Subramaniam, V. Normand, Determination of covalent cross-linker efficacy of gelatin strands using calorimetric analyses of the gel state, Soft Matter. 7 (2011) 3315-3322.

[59] X. Wu, Y. Liu, X. Li, P. Wen, Y. Zhang, Y. Long, X. Wang, Y. Guo, F. Xing, J. Gao, Preparation of aligned porous gelatin scaffolds by unidirectional freeze-drying method, Acta Biomater. 6 (2010) 1167-1177.

[60] B. Chiou, R.J. Avena-Bustillos, P.J. Bechtel, H. Jafri, R. Narayan, S.H. Imam, G.M. Glenn, W.J. Orts, Cold water fish gelatin films: Effects of cross-linking on thermal, mechanical, barrier, and biodegradation properties, Eur. Polym. J. 44 (2008) 3748-3753.

[61] X. Liu, Y. Won, P.X. Ma, Porogen-induced surface modification of nano-fibrous poly(1lactic acid) scaffolds for tissue engineering, Biomaterials. 27 (2006) 3980-3987.

[62] J.F. Martucci, R.A. Ruseckaite, A. Vázquez, Creep of glutaraldehyde-crosslinked gelatin films, Mat. Sci. Eng. A. 435-436 (2006) 681-686.

[63] A. Bigi, G. Cojazzi, S. Panzavolta, N. Roveri, K. Rubini, Stabilization of gelatin films by crosslinking with genipin, Biomaterials. 23 (2002) 4827-4832.

[64] P. Gentile, V. Chiono, C. Tonda-Turo, C. Mattu, F. Baino, C. Vitale-Brovarone, G. Ciardelli, Bioresorbable glass effect on the physico-chemical properties of bilayered scaffolds for osteochondral regeneration, Mater Lett. 89 (2012) 74-76. 


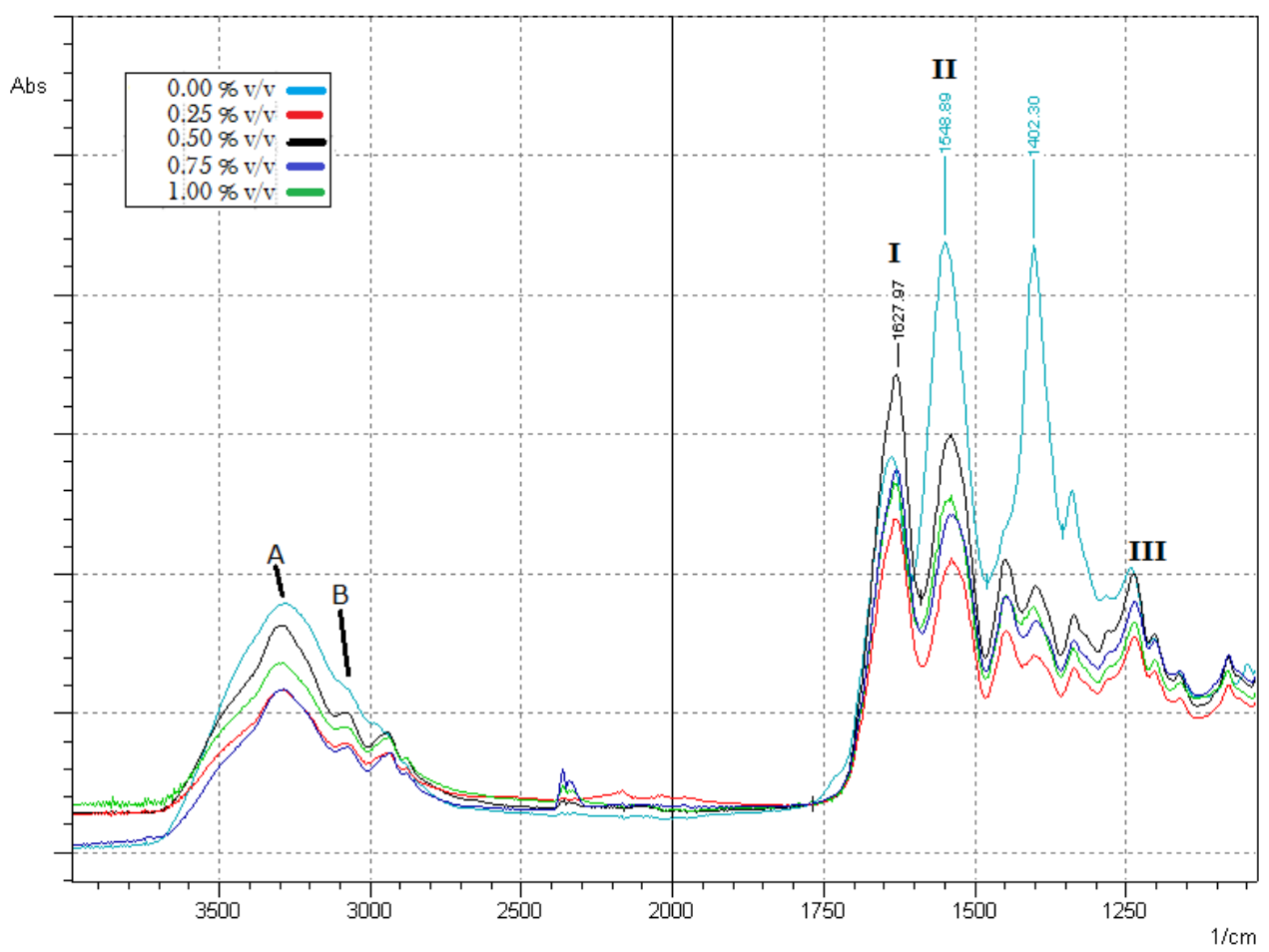

Figure 1: FT-IR spectra of scaffolds crosslinked with $0,0.25,0.50,0.75$, and $1.00 \%$ v/v GTA aqueous solutions are shown. Amide I and III bands of the crosslinked samples showed a slight shift towards a lower wave number when compared with the non-crosslinked sample. Amide I, II, III, A, and B are marked for indication. 

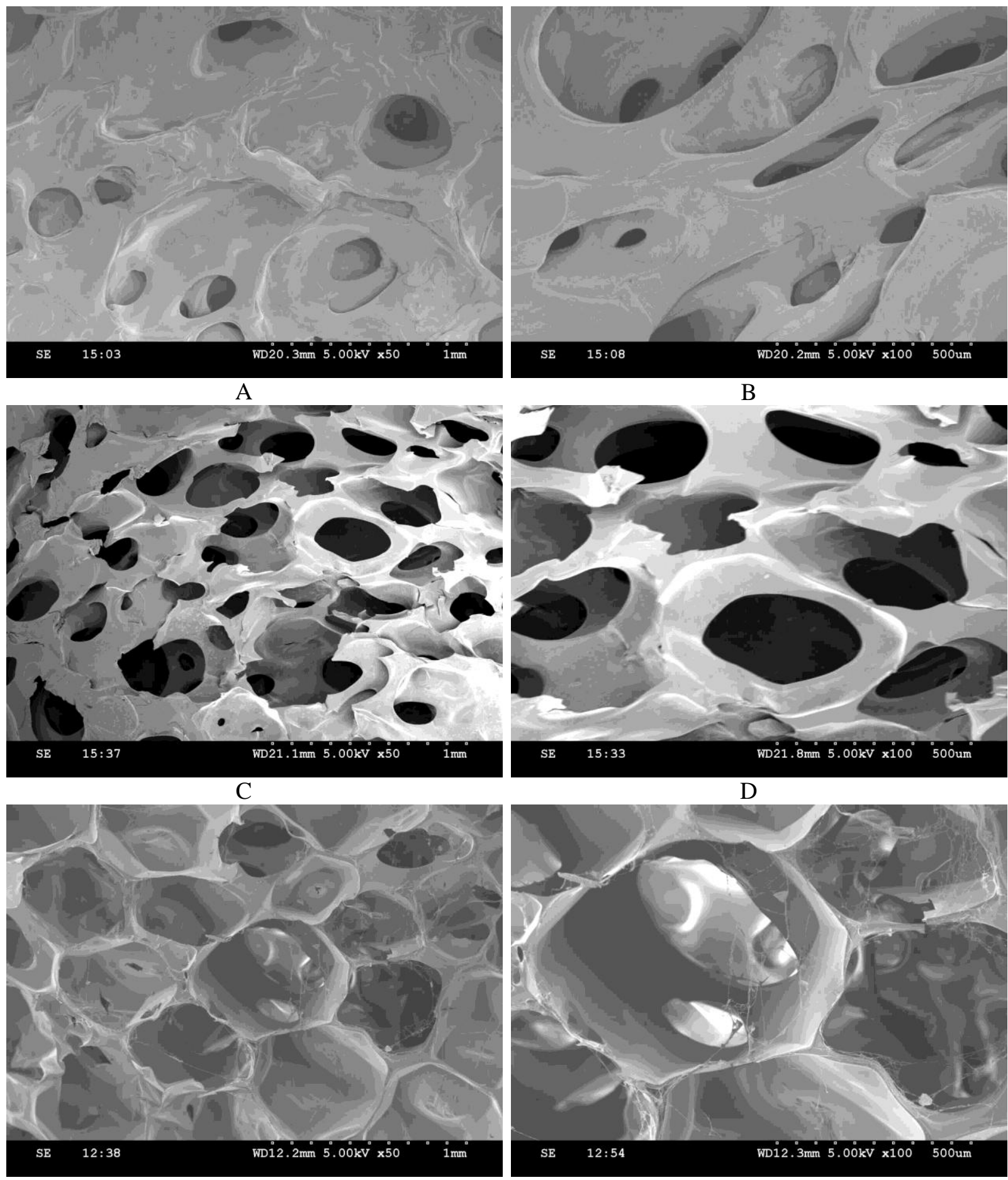

E

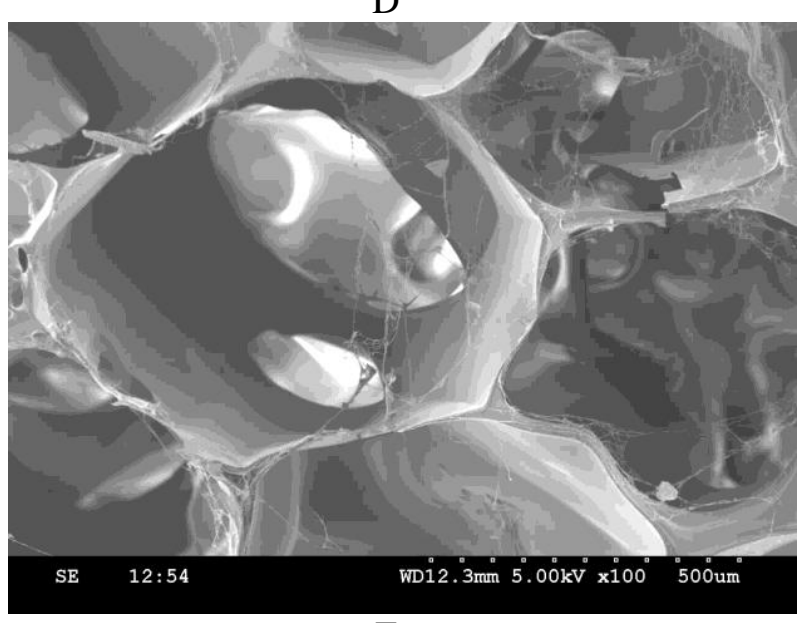

F

Figure 2: SEM images showing interconnectivity and the pore structure of gelatin scaffolds as the concentration of GTA increased from 0 to $1.00 \%$ v/v. (A) and (B) non-crosslinked samples both at 50x and 100x magnification, respectively, (C) and (D) scaffolds crosslinked with $0.50 \%$ v/v GTA solution, at 50x and 100x magnification; (E) and (F), scaffolds crosslinked with $1.00 \% \mathrm{v} / \mathrm{v}$ GTA solution at 50x and 100x magnification, respectively 


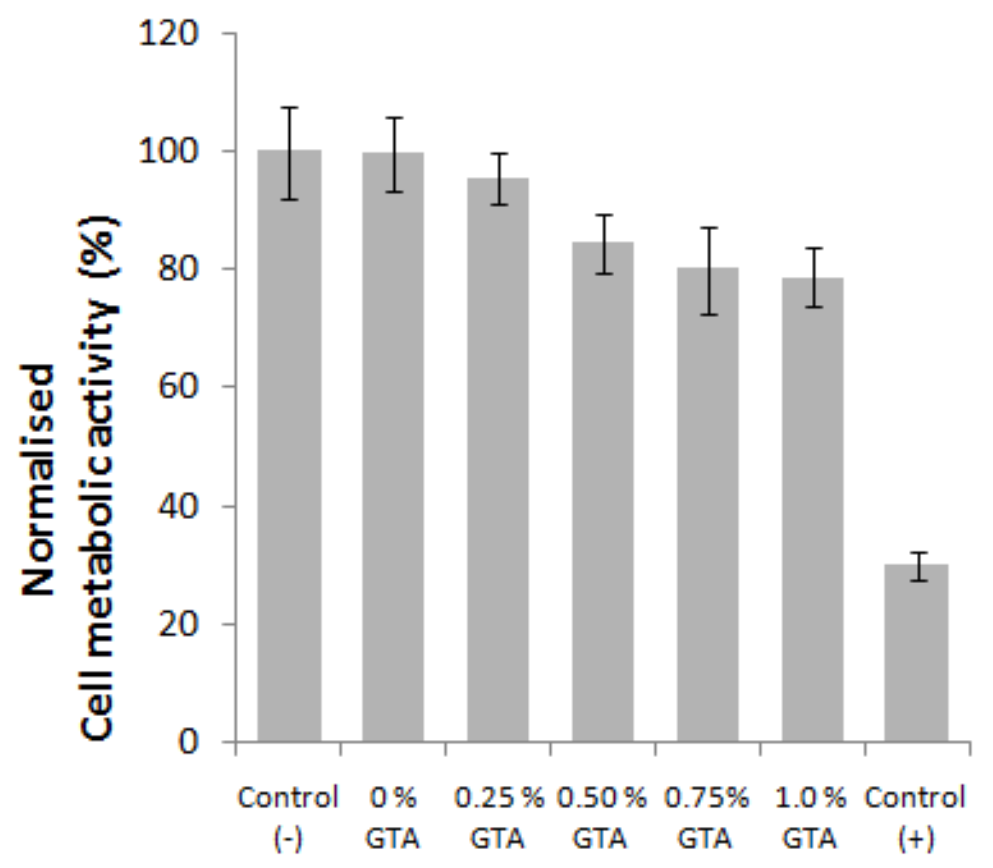

Figure 3: Normalised metabolic activity of cells in contact with scaffolds crosslinked at different concentrations of GTA. Results are shown as average \pm standard deviation $(n=3)$. 


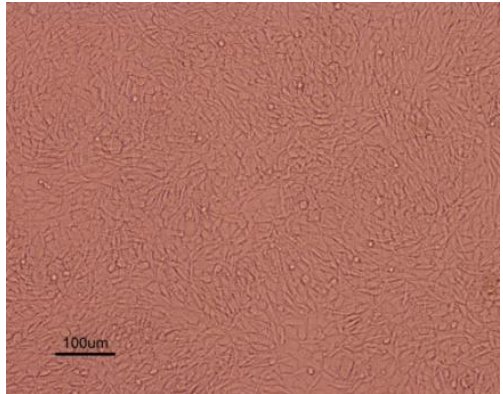

A

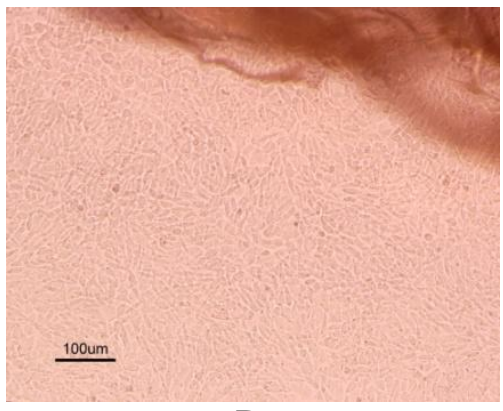

$\mathrm{D}$

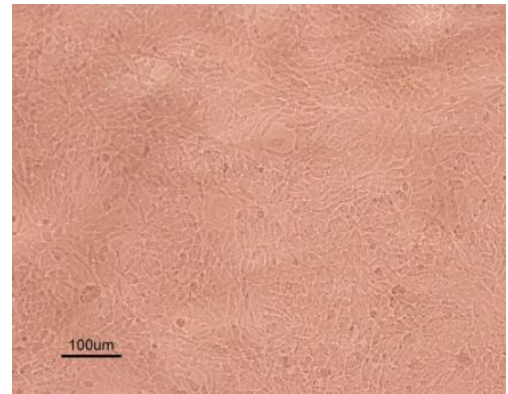

$\mathrm{B}$

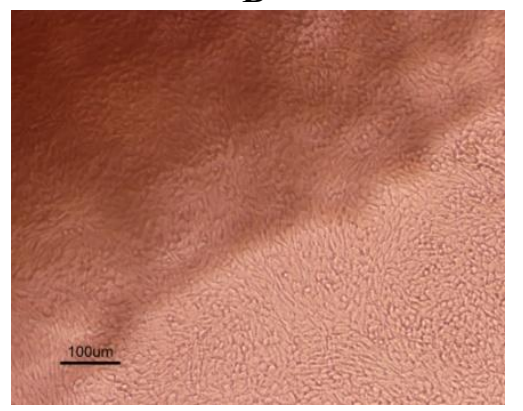

$\mathrm{E}$

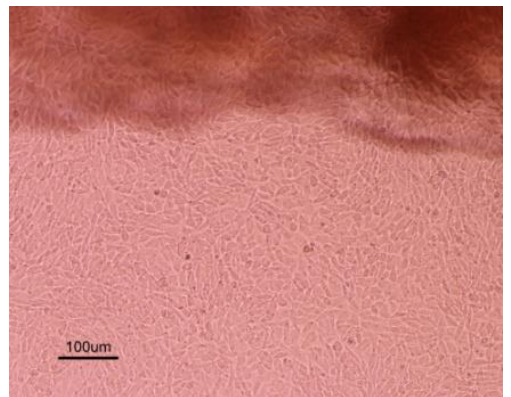

$\mathrm{C}$

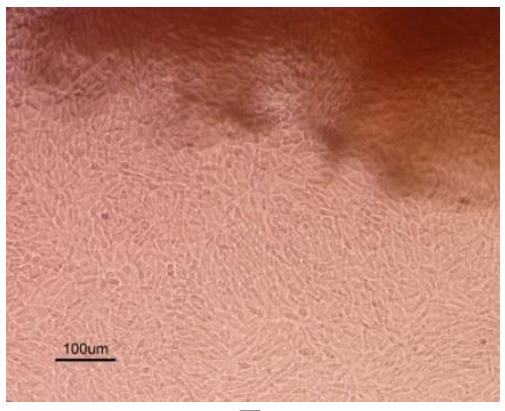

$\mathrm{F}$

Figure 4: The results of direct contact tests at 100X magnification are shown: Negative control (without scaffold) (A), Non-crosslinked scaffold (B), scaffold crosslinked with 0.25\% GTA (C), 0.50\% GTA (D), 0.75\% GTA (E), and $1.00 \%$ GTA (F). Scaffolds were placed above the cell culture and observed with an inverted microscope for the direct contact tests. Scale bar represents $100 \mu \mathrm{m}$. 
Table 1: Thermal analysis of porous gelatin scaffolds crosslinked at different concentrations of GTA. Peak temperature is designated as $T_{d}$ in this study. Results are shown as an average \pm standard deviation $(n=3)$.

\begin{tabular}{|c|c|c|}
\hline $\begin{array}{c}\text { GTA concentration } \\
(\mathbf{\%} \mathbf{v} / \mathbf{v})\end{array}$ & $\begin{array}{c}\text { Denaturation Temperature }\left(\mathbf{T}_{\mathbf{d}}\right) \\
\left({ }^{\circ} \mathbf{C}\right)\end{array}$ & $\begin{array}{c}\text { Enthalpy of Transition } \\
(\mathbf{J} / \mathbf{g})\end{array}$ \\
\hline 0 & $43.97( \pm 1.6)$ & $-14.22( \pm 1.4)$ \\
\hline 0.25 & $77.45( \pm 0.3)$ & $-13.71( \pm 0.3)$ \\
\hline 0.50 & $73.52( \pm 2.3)$ & $-12.04( \pm 0.3)$ \\
\hline 0.75 & $77.94( \pm 0.9)$ & $-10.57( \pm 1.2)$ \\
\hline 1.00 & $76.25( \pm 2.3)$ & $-9.65( \pm 0.9)$ \\
\hline
\end{tabular}


Table 2: Compression strength of the gelatin scaffolds. Results are shown as an average \pm standard deviation $(n=3)$.

\begin{tabular}{|c|c|}
\hline $\begin{array}{c}\text { GTA Concentration } \\
(\boldsymbol{\%} \mathbf{v} / \mathbf{v})\end{array}$ & $\begin{array}{c}\text { Compression Strength } \\
(\mathbf{k P a})\end{array}$ \\
\hline 0 & $2.77( \pm 0.75)$ \\
\hline 0.25 & $3.34( \pm 0.91)$ \\
\hline 0.50 & $3.58( \pm 1.01)$ \\
\hline 0.75 & $6.34( \pm 1.48)$ \\
\hline 1.00 & $8.53( \pm 3.12)$ \\
\hline
\end{tabular}


Table 3: Tensile properties of the prepared gelatin scaffolds. The tensile strain of the scaffolds is reduced as a result of crosslinking. Results shown as an average \pm standard deviation $(n=3)$.

\begin{tabular}{|c|c|c|c|}
\hline $\begin{array}{c}\text { GTA Concentration } \\
(\% \mathbf{v} / \mathbf{v})\end{array}$ & Tensile Strength $(\mathbf{k P a})$ & Elastic Modulus (kPa) & Tensile Strain (\%) \\
\hline 0 & $1.09( \pm 0.31)$ & $0.94( \pm 0.30)$ & $53.43( \pm 18.00)$ \\
\hline 0.25 & $4.14( \pm 0.65)$ & $2.67( \pm 1.31)$ & $231.15( \pm 43.90)$ \\
\hline 0.50 & $5.37( \pm 2.07)$ & $12.18( \pm 2.01)$ & $158.91( \pm 54.21)$ \\
\hline 0.75 & $4.33( \pm 1.00)$ & $13.09( \pm 5.18)$ & $102.83( \pm 40.36)$ \\
\hline 1.00 & $3.80( \pm 0.69)$ & $7.98( \pm 2.15)$ & $39.59( \pm 16.03)$ \\
\hline
\end{tabular}

